\title{
Towards a General Fuzzy Ontology and Its Construction
}

\author{
Huamao Gu ${ }^{1,2}$ Hexin $\mathrm{Lv}^{3}$ Ji Gao ${ }^{1}$ Jinqin Shi ${ }^{2}$ \\ ${ }^{1}$ College of Computer Science, Zhejiang University, Hangzhou 310027, P.R. China \\ ${ }^{2}$ Sensory Sci. Lab, Zhejiang Gongshang University, Hangzhou 310018, P.R. China \\ ${ }^{3}$ College of Information, Zhejiang Shuren University, Hangzhou 310015, P.R. China
}

\begin{abstract}
Most of the current fuzzy ontologies are too domainspecific and don't support reasoning on fuzzy descriptions. By investigating the uncertainty information in the real world, we discover three widely existing general fuzzy relations. Based on such investigation and motivated by the desire to support reasoning and guide fuzzy ontology construction, we propose a reasoning-enabled general fuzzy ontology including these general fuzzy relations. To support such ontology in theory, we introduce a fuzzy description logic ef-SHIN, and define its syntax and semantics. Besides, a reference process for guiding the construction of fuzzy concept base relations in fuzzy ontology is also presented.
\end{abstract}

Keywords: Fuzzy ontology, Fuzzy description logic

\section{Introduction}

Ontology has recently been getting increasingly popular in many areas, especially in Semantic Web community. Nowadays, ontology has been conceived as a good mechanism to describe the sharable and common understanding of domain knowledge. In knowledge engineering, ontology informally consists of a hierarchical description of concepts, along with the description of the properties of concepts, and probably with other ingredients. These concepts and properties are usually treated as crisp sets in implementation. We know, however, the real world is full of uncertainties, and modeling as such just approximates to and is not exactly consistent with the real world.

During the application of ontology, more and more practitioners realized the difficulty in describing uncertainty knowledge in ontology. Therefore, some pioneers incorporated fuzzy logic into ontology. Lee et al. [2] presented a four-layered fuzzy ontology and applied it to news summarization. Quan et al. [5] provided a fuzzy ontology generation framework for generating concept hierarchy and presented a fuzzy scholarly ontology.

From the present fuzzy ontology applications, we can see that most fuzzy ontologies are domain-specific and just simply integrate some specific fuzzy relations into ordinary ontologies. As such, they make little sense in directing the construction of fuzzy ontologies in other domains. Another drawback of the present fuzzy ontologies is that they don't support reasoning services on fuzzy descriptions. So the uncertainty information has not been made full use of in current fuzzy ontologies. However, there is no research on the general fuzzy ontology that is not restricted to certain domain and support reasoning services so far. To improve this situation, we focus on a reasoningenabled general fuzzy ontology independent of specific domains from theoretical and practical perspectives.

As the theoretical counterpart of fuzzy ontology, the fuzzy description logics (FDLs) have also attracted much attention from researchers. For example, Straccia [6] presented fuzzy ALC and an algorithm for reasoning. Lu et al. [3] proposed an extended fuzzy ALCN and designed tableau algorithms for that. Stoilos et al. [8] introduced a tableaux algorithm for the fuzzy extensions of $S \mathcal{H} I \mathcal{N}$. Straccia [7] presented fuzzy $S \mathcal{H} O I \mathcal{N}(\mathcal{D})$ for a fuzzy OWL DL. Kang et al. [4] integrated comparison expressions into fuzzy description logic and discussed the reasoning in this issue. All these efforts provide a good foundation for fuzzy ontology construction and reasoning.

In this paper, we propose a reasoning-enabled general fuzzy ontology based on the observation that three general fuzzy relations exist widely among the real world. And we formally provide a theoretical analysis of it by extending the $f_{\mathcal{L D}}-S \mathcal{H} I \mathcal{N}$ [7] with these three general fuzzy relations. To make it more practical, we also introduce a reference process for building these general fuzzy relations in fuzzy ontology applications.

\section{Fuzzy set theory}


Fuzzy sets were first introduced by Zadeh [9] as a way to deal with vague concepts like baldhead, low speed and the like. To make the paper self-contained, we give a minimalist survival guide to fuzzy set theory before further discussing the general fuzzy ontology. Now, we formally give some basic definitions in fuzzy set theory.

Definition 1. A fuzzy set A with respect to a universe of discourse $\mathrm{X}$ is characterized by a membership function $\mu_{\mathrm{A}}: \mathrm{X} \rightarrow[0,1]$, assigning an A-membership degree, $\mu_{\mathrm{A}}(\mathrm{x})$, to each element $\mathrm{x}$ in $\mathrm{X}$. Membership function $\mu_{\mathrm{A}}(\mathrm{x})$ provides a measure of the degree of the belonging of $x$ to $A$. Typically, if $\mu_{A}(x)=1$ then $x$ definitely belongs to $\mathrm{A}$, while $\mu_{\mathrm{A}}(\mathrm{x})=0.7$ means that $\mathrm{x}$ is "likely" to be an element of A by a degree of 0.7 . For ease of representation, a fuzzy set A over universe $\mathrm{X}$ is organized into a set of ordered pairs:

$$
\mathrm{A} \equiv\left\{\left(\mathrm{x}, \mu_{\mathrm{A}}(\mathrm{x})\right) \mid \mathrm{x} \in \mathrm{X}\right\} \text {. }
$$

Definition 2. Let $X$ and $Y$ be two universes of discourse. A (binary) fuzzy relation $\mathrm{R}$ in the product space $X \times Y$ is a membership function $R: X \times Y \rightarrow[0,1]$, i.e. $R(X, Y) \equiv\left\{\left((x, y), \mu_{R}(x, y)\right) \mid(x, y) \in X \times Y\right\}$. In the context of fuzzy sets, a fuzzy relation over two universes $\mathrm{X}$ and $\mathrm{Y}$ is a fuzzy set in the product space $\mathrm{X} \times \mathrm{Y}$. Indeed, fuzzy relation represents a degree of presence or absence of association, interaction, or interconnectedness between the elements of two or more crisp sets.

Definition 3. Let $X$ and $Y$ be both finite sets, $\mathrm{X}=\left\{\mathrm{x}_{1}, \mathrm{x}_{2}, \ldots, \mathrm{x}_{\mathrm{m}}\right\}$ and $\mathrm{Y}=\left\{\mathrm{y}_{1}, \mathrm{y}_{2}, \ldots, \mathrm{y}_{\mathrm{n}}\right\}$. Then a fuzzy relation over $\mathrm{X}$ and $\mathrm{Y}$ can be represented as an $\mathrm{m}-\mathrm{by}-\mathrm{n}$ matrix $R=\left[r_{i j}\right]_{m \times n}: r_{i j}=\mu{ }_{R}\left(x_{i}, y_{j}\right) \quad(i=1,2, \ldots, m$; $\mathrm{j}=1,2, \ldots, n)$. Such matrix is called fuzzy matrix. Obviously, fuzzy matrix is just another format of fuzzy relation.

\section{A general fuzzy ontology}

In traditional ontology theory, concepts and roles are crisp sets. Nevertheless, there is too much fuzziness in the real world. Thus, there is no exact consistency between the modeling of ontology and the real world. To narrow the gap, some practitioners have already developed different domain-specific fuzzy ontologies. But these fuzzy ontologies don't support reasoning on fuzzy descriptions and have little meaning for guiding fuzzy ontology applications in other domains. We go further with an inside study in the fuzziness in ontology, giving birth to a reasoning-enabled general fuzzy ontology independent of specific domains.

After an in-depth investigation of uncertainty information in ontology, we discover three general fuzzy relations widely existing in the modeling of domain knowledge, which play crucial roles in precisely modeling domain knowledge. Definitions 4 to 6 give formal descriptions of these three relations.

\subsection{Three general fuzzy relations}

Before going ahead, we introduce two basic relations in ontology, "kind-of" ( $\mathrm{R}_{\mathrm{kf}}$ for short) and "instance-of" ( $\mathrm{R}_{\text {if }}$ for short). Relation "kind-of" consists of those pairs of concepts with one being more particular than the other, while "instance-of" associates each individual with its concept.

Definition 4. Supposing that X, Y are two concepts in an ontology, a fuzzy instance relation (FIR for short) $\mathrm{R}$ between $\mathrm{X}$ and $\mathrm{Y}$ is a fuzzy relation, i.e., $\mathrm{R} \equiv\{((\mathrm{x}, \mathrm{y})$, $\left.\left.\mu_{\mathrm{R}}(\mathrm{x}, \mathrm{y})\right) \quad(\mathrm{x}, \mathrm{X}), \quad(\mathrm{y}, \mathrm{Y}) \in \mathrm{R}_{\mathrm{if}}\right\} . \quad$ As obvious, FIR corresponds to an ordinary role in fuzzy description logic. So, there is no need for any extensions to implementation such relation in fuzzy ontology.

Definition 5. The occurring of fuzzy concept relation (FCR for short) is based on the observation that there are some special fuzzy instance relations in which all the membership degrees between the instances of two concepts are the same. So it is just a special kind of fuzzy instance relation. For example, supposing A and $\mathrm{B}$ are two concepts, there is a fuzzy instance relation $\mathrm{R}$ $\equiv\left\{\left((\mathrm{a}, \mathrm{b}), \mu_{\mathrm{R}}(\mathrm{a}, \mathrm{b})\right) \mid(\mathrm{a}, \mathrm{A}),(\mathrm{b}, \mathrm{B}) \in \mathrm{R}_{\text {if }}\right\}$. If $\mu_{\mathrm{R}}(\mathrm{a}, \mathrm{b})$ is always the same, say 0.6 , for all the $\mathrm{a}$ in $\mathrm{A}$ and $\mathrm{b}$ in $\mathrm{B}$, we call $\mathrm{R}$ a fuzzy concept relation, as shown in Fig. 1. Fuzzy concept relation can also be represented simply by a triple $<\mathrm{X}, \mathrm{Y}, \mu_{\mathrm{R}}(\mathrm{X}, \mathrm{Y})>$ where $\mu_{\mathrm{R}}(\mathrm{X}, \mathrm{Y})$ is a value in close interval $(0,1]$ which means the strength of association between $\mathrm{X}$ and $\mathrm{Y}$ in certain aspect. To make the format be in consistency with FCBR in definition 6, we prefer to represent a FCR with a set $\left\{<\mathrm{X}, \mathrm{Y}, \mu_{\mathrm{R}}(\mathrm{X}, \mathrm{Y})>\right\}$ that has only one element.

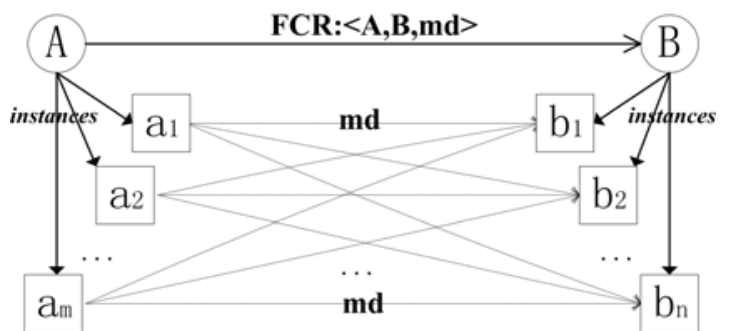

Fig. 1: A FCR relation between concepts $\mathrm{A}$ and $\mathrm{B}$ with a membership degree of $m d$. Circles designate concepts, while squares denote individuals. Dotted lines mean associations between individuals with a same membership degree $m d$.

Definition 6. Fuzzy concept base relation (FCBR for short) is a set of fuzzy concept relations. Supposing that $\mathrm{X}, \mathrm{Y}$ are two concepts, there are derived concepts $\left\{\mathrm{X}_{1}, \mathrm{X}_{2} \ldots, \mathrm{X}_{\mathrm{m}}\right\}$ of $\mathrm{X}$ and $\left\{\mathrm{Y}_{1}, \mathrm{Y}_{2} \ldots, \mathrm{Y}_{\mathrm{n}}\right\}$ of $\mathrm{Y}$, namely, there is "kind-of" relation between $X_{i}$ and $X$, and so does between $Y_{i}$ and $Y$. If there are fuzzy concept relations $<\mathrm{X}_{\mathrm{i}}, \mathrm{Y}_{\mathrm{j}}, \mu_{\mathrm{R}}\left(\mathrm{X}_{\mathrm{i}}, \mathrm{Y}_{\mathrm{j}}\right)>$ for all pairs $\left(\mathrm{X}_{\mathrm{i}}, \mathrm{Y}_{\mathrm{j}}\right)$ where 
$1 \leqslant \mathrm{i} \leqslant \mathrm{m}$ and $1 \leqslant \mathrm{j} \leqslant \mathrm{n}$, we call that there is a fuzzy concept base relation $\mathrm{R}$ on $\mathrm{X}$ and $\mathrm{Y}$, as shown in Fig. 2. Fuzzy concept base relation can be represented by a set of triples $\left\{<\mathrm{X}_{\mathrm{i}}, \mathrm{Y}_{\mathrm{j}}, \mu_{\mathrm{R}}\left(\mathrm{X}_{\mathrm{i}}, \mathrm{Y}_{\mathrm{j}}\right)>\mid\left(\mathrm{X}_{\mathrm{i}}, \mathrm{X}\right),\left(\mathrm{Y}_{\mathrm{j}}, \mathrm{Y}\right)\right.$ $\left.\in \mathrm{R}_{\mathrm{kf}}\right\}$, namely a group of FCRs. The fuzzy concept base relation originates from our experience in domain ontology construction.

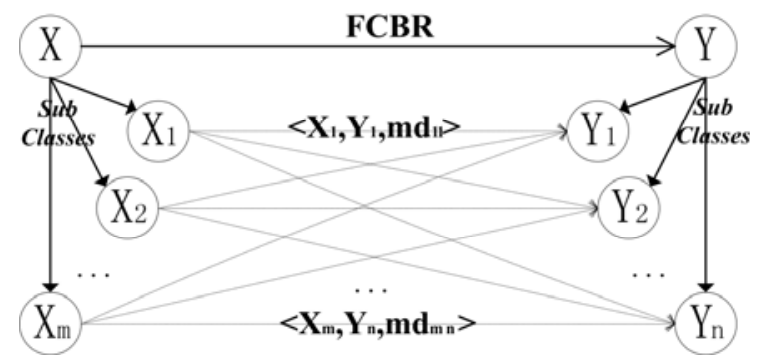

Fig. 2: A FCBR relation between super-concepts $\mathrm{X}$ and $\mathrm{Y}$, which consists of a group of FCR relations. Circles designate concepts. A dotted line means an association between two concepts with a membership degree $\mathrm{md}_{\mathrm{ij}}$.

\subsection{General fuzzy ontology based on ef-SHIN}

Now, it comes to the issue of how to put these three general fuzzy relations into use in ontology. Firstly, we will draw an analysis in a description logic view. As these three fuzzy relations do not touch upon concrete data types (the concrete domain) and named individuals (nominals), we base the general fuzzy ontology on an extended version of $f_{K D}-S \mathcal{H} I \mathcal{N}$ discussed in [8] for the sake of simplicity. And because of the above features, three general fuzzy relations can be easily integrated into other forms of FDLs. From now on, we will refer to the extended version as ef-SHITN.

Alphabeths. Let $C N, R N_{n}, R N_{c}$, and $I N$ be non-empty finite and pair-wise disjoint set of concept names, ordinary role names, FCR and FCBR role names, and individual names. Note that elements in $R N_{c}$ can't be used to build ef-SHISN-concepts directly. We assume that there is a counterpart in $R N_{n}$ for each element $e$ in $R N_{c}$, denoted as $\operatorname{Cpt}(e)$. All the counterparts of the elements in $R N_{c}$ compose the $\operatorname{Cpt}\left(R N_{c}\right)$ which can be viewed as ordinary role names. In implementation, the FCR/FCBR role names and their counterparts in $R N_{n}$ can take the same names.

Roles. Let $\mathrm{Rn} \in R N_{n}$ be a role name, an $e f-S \mathcal{H I N}$-role $R$ is defined by the abstract syntax: $R::=\mathrm{Rn} \mid R^{-}$. Note that an $e f-S \mathcal{H} I \mathcal{N}$-role corresponds to a fuzzy instance relation. The inverse relation of roles is symmetric, and to avoid considering roles such as $\mathrm{R}^{--}$, we define a function Inv, which returns the inverse of a role, more precisely $\operatorname{Inv}(\mathrm{R}):=\mathrm{Rn}^{-}$if $\mathrm{R}=\mathrm{Rn}$ and $\operatorname{Inv}(\mathrm{R}):=\mathrm{Rn}$ if $\mathrm{R}=\mathrm{Rn}^{-}$.

Concepts. The set of ef-SHIN -concepts is defined by the following syntactic rules, where $\mathrm{A} \in C N$ is a concept name, C, D are two ef-SHIN -concepts,

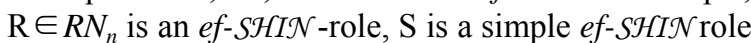
[1], $\mathrm{R}_{\mathrm{c}} \in R N_{c}$ is a FCR/FCBR role and $\mathrm{n} \in \mathbb{N}$ :

$$
\begin{aligned}
& \mathrm{C} \rightarrow \mathrm{T}|\perp| \mathrm{A}|\mathrm{C} \cup \mathrm{D}| \mathrm{C} \cap \mathrm{D}|\neg \mathrm{C}| \forall \mathrm{R} . \mathrm{C}|\exists \mathrm{R} . \mathrm{C}| \\
& (\geqslant \mathrm{n} \mathrm{S})|(\leqslant \mathrm{n} \mathrm{S})| \forall \mathrm{Cpt}\left(\mathrm{R}_{\mathrm{c}}\right) . \mathrm{C} \mid \exists \mathrm{Cpt}\left(\mathrm{R}_{\mathrm{c}}\right) . \mathrm{C}
\end{aligned}
$$

TBox. A fuzzy TBox $\mathcal{T}$ consists of a finite set of fuzzy concept axioms of the form $\mathrm{C} \subseteq \mathrm{D}$ and of the form $\mathrm{C} \equiv \mathrm{D}$, where $\mathrm{C}$ is a concept name and $\mathrm{D}$ is a concept.

RBox. A fuzzy RBox $\boldsymbol{R}$ consists of a finite set of fuzzy transitivity role axioms Trans $(R n)$ where $R n$ is a role name, and fuzzy inclusion axioms $\mathrm{R} \sqsubseteq \mathrm{S}$ where $\mathrm{R}$ and $\mathrm{S}$ are ef-SHIN roles. The reflexive-transitive closure of the role inclusion relationship is denoted with $\Xi^{*}$. A role not having transitive sub-roles is called simple role.

ABox. A fuzzy ABox $\mathcal{A}$ consists of a finite set of fuzzy assertions. A fuzzy assertion (Straccia, 2001) is of the form $<$ a: $\mathrm{C} \bowtie \mathrm{n}>,<(\mathrm{a}, \mathrm{b}): \mathrm{R} \bowtie \mathrm{n}>$, where $\mathrm{n} \in[0,1]$ and $\bowtie$ stands for $\geq,>, \leq$ or $<$ or $\mathrm{a} \neq \mathrm{b}$, for $\mathrm{a}, \mathrm{b} \in I N$.

General Fuzzy Ontology. A general fuzzy ontology $\mathbf{G}$ based on ef-SHIN is a triple $\langle\mathcal{T}, \mathcal{R}, \mathcal{A}\rangle$ where $\mathcal{T}$ is a fuzzy TBox, $R$ is a fuzzy RBox and $\mathcal{A}$ is a fuzzy ABox.

\section{Semantics}

The semantics of fuzzy DLs are provided by a fuzzy interpretation [6]. A fuzzy interpretation of the general fuzzy ontology is a pair $I=\left(C N \cup \Delta^{I}, \cdot{ }^{I}\right)$ consisting of a union of a non empty set $\Delta^{I}$ (called the domain) and $C N$, and of a fuzzy interpretation function - I that assigns to each concept name $\mathrm{A} \in C N$ a function $\mathrm{A}^{I}: \Delta^{I} \rightarrow[0,1]$, to each ordinary role name $\mathrm{R}$ $\in R N_{n}$ a function $\mathrm{R}^{I}: \Delta^{I} \times \Delta^{I} \rightarrow[0,1]$, to each FCR and FCBR role name $\mathrm{R}_{\mathrm{c}} \in R N_{c}$ a function $\mathrm{R}_{\mathrm{c}}{ }^{I}: C N \times$ $C N \rightarrow[0,1]$, and to each individual $\mathrm{a} \in \mathrm{IN}$ an element in $\Delta^{I}$. The mapping ${ }^{-}{ }^{I}$ is extended to concepts and roles as specified in Table 1 (where $\mathrm{a}, \mathrm{b} \in \Delta^{I}$ ):

$\top^{I}(\mathrm{a})=1$

$\perp^{I}$ (a) $=0$

$(\neg \mathrm{C})^{I}(\mathrm{a})=1-\mathrm{C}^{I}(\mathrm{a})$

$\left(\mathrm{R}^{-}\right)^{I}(\mathrm{~b}, \mathrm{a})=\mathrm{R}^{\mathrm{I}}(\mathrm{a}, \mathrm{b})$

$(\mathrm{C} \cup \mathrm{D})^{I}(\mathrm{a})=\max \left(\mathrm{C}^{I}(\mathrm{a}), \mathrm{D}^{I}(\mathrm{a})\right)$

$(\mathrm{C} \cap \mathrm{D})^{I}(\mathrm{a})=\min \left(\mathrm{C}^{I}(\mathrm{a}), \mathrm{D}^{I}(\mathrm{a})\right)$

$(\forall \text { R.C })^{I}(\mathrm{a})=\inf _{\mathrm{b} \in} \Delta^{I}\left\{\max \left(1-\mathrm{R}^{I}(\mathrm{a}, \mathrm{b}), \mathrm{C}^{I}(\mathrm{~b})\right)\right\}$

$(\exists \text { R.C })^{I}(\mathrm{a})=\sup _{\mathrm{b} \in} \Delta^{I}\left\{\min \left(\mathrm{R}^{I}(\mathrm{a}, \mathrm{b}), \mathrm{C}^{I}(\mathrm{~b})\right)\right\}$

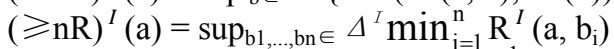

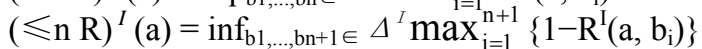

$\operatorname{Cpt}\left(\mathrm{R}_{\mathrm{c}}\right)^{I}(\mathrm{a}, \mathrm{b})=\sup _{\mathrm{X}, \mathrm{Y} \in \mathrm{CN}}\left\{\min \left(\mathrm{f}_{\mathrm{m}}\left(\min \left(\mathrm{X}^{I}(\mathrm{a}), \mathrm{Y}^{I}(\mathrm{~b})\right)\right)\right.\right.$,

$$
\left.\left.\mathrm{R}_{\mathrm{c}}^{I}(\mathrm{X}, \mathrm{Y})\right)\right\}
$$




$$
\begin{aligned}
\left(\forall \mathrm{Cpt}\left(\mathrm{R}_{\mathrm{c}}\right) \cdot \mathrm{C}\right)^{I}(\mathrm{a})=\inf _{\mathrm{b} \in \Delta^{I}\left\{\operatorname { m a x } \left(1-\mathrm{Cpt}\left(\mathrm{R}_{\mathrm{c}}\right)^{I}(\mathrm{a}, \mathrm{b}),\right.\right.} & \left.\left.\mathrm{C}^{I}(\mathrm{~b})\right)\right\} \\
\left(\exists \mathrm{Cpt}\left(\mathrm{R}_{\mathrm{c}}\right) \cdot \mathrm{C}\right)^{I}(\mathrm{a})= & \left.\left.\sup _{\mathrm{b} \in \Delta^{I}\left\{\operatorname { m i n } \left(\operatorname{Cpt}\left(\mathrm{R}_{\mathrm{c}}\right)^{I}(\mathrm{a}, \mathrm{b}),\right.\right.} \mathrm{C}^{I}(\mathrm{~b})\right)\right\}
\end{aligned}
$$

Table 1: Semantics of ef-SHIN - concepts.

Here, we just comment briefly the semantics of $\operatorname{Cpt}\left(\mathrm{R}_{\mathrm{c}}\right)^{I}(\mathrm{a}, \mathrm{b})$, for more detail, see [8]. Function $f_{m}$ is defined on closed interval $[0,1]$ as (see Fig. 3):

$$
f_{m}(x)= \begin{cases}0 & (0 \leq x<m) \\ m & (m \leq x \leq 1)\end{cases}
$$

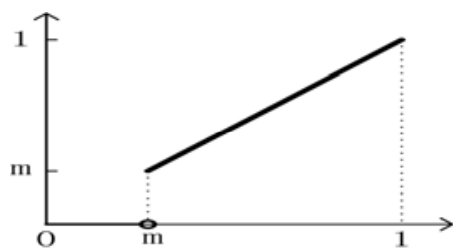

Fig. 3: Function $f_{m}$.

Parameter $m$ is a critical value determining for $\mathrm{X}^{I}(a)$ and $\mathrm{Y}^{I}(b)$ the possibility of taking part in calculation of $\operatorname{Cpt}\left(\mathrm{R}_{\mathrm{c}}\right)^{I}(a, b)$. Function $f_{m}$ leaves out most of the items in which $a$ (or $b$ ) belongs to X (or Y) with low membership degree (i.e. less than $m$ ). That means that those elements with low membership degrees to certain concepts are treated as definitely not belonging to them. And this is consistent with the real world.

\section{Reasoning}

Reasoning plays a pivotal role in most ontology-based applications. As known, description logic is the underlying theoretical counterpart of ontology. In description logics, most of the inference services can be reduced to the problem of consistence checking for Aboxes. And consistency is usually checked with tableaux algorithms. Stoilos [8] provided a fuzzy tableaux algorithm for checking consistency of $f_{K D^{-}}$ SHIN based knowledge base.

From the description above, we have seen that each element $\mathrm{R}_{\mathrm{c}}$ in $R N_{c}$ only takes part in the calculation of the membership degree of its counterpart $\operatorname{Cpt}\left(\mathrm{R}_{\mathrm{c}}\right)$ over $\Delta^{I}$ and has no influence on other factors. While that of the ordinary ef-SHIN role (namely FIR) usually adopts some membership functions or domain experts' suggestions. Therefore, the FCR or FCBR roles don't intervene in the inference services directly. In this respect, the ef-SHIN in this paper can be thought of as the same as $f_{K D^{-}}$ $S \mathcal{H I N}$ [8]. So the fuzzy tableaux algorithm [8] can be also applied to reasoning on the general fuzzy ontology without any modification. Besides, we have also exploited out another fuzzy tableaux algorithm of reasoning on the general fuzzy ontology directly, which will appear in another paper as the second of a pair in the same issue.

\section{Reference processes for construction}

In this section, we just provide a reference process to guide the building of fuzzy relations of FCBR, instead of a full-fledged methodology for fuzzy ontology construction. See Fig. 4 for more details. This step acts as the specifying the interpretation function $\mathrm{R}_{\mathrm{c}}{ }^{I}: C N \times$ $\mathrm{CN} \rightarrow[0,1]$ for $\mathrm{FCR} / \mathrm{FCBR}$

Definition 7. Fuzzy Relation Tag. Supposing $X$ and $\mathrm{Y}$ are two concepts, there is a triple $<\mathrm{X}, \mathrm{Y}, \mathrm{N}\rangle$ where $\mathrm{N}$ is a character string with the value of 'FCBR', 'FCR' or 'FIR' which means there is a relation of type $\mathrm{N}$ on $\mathrm{X}$ and $\mathrm{Y}$. This triple is called a fuzzy relation tag.

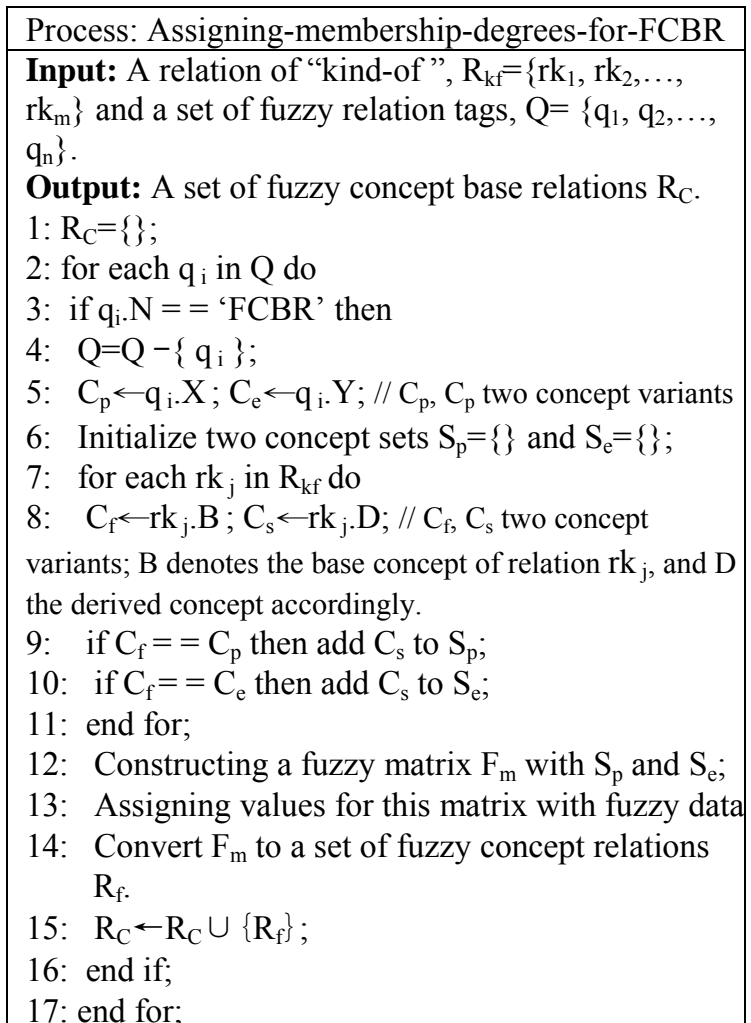

Fig. 4: Process of assigning degrees for FCBR.

As for adding degrees for fuzzy concept relation, we suggest to group the entire fuzzy concept relations into several equivalence classes so that the assigning could be more efficient. And assigning degrees to fuzzy instance relations is very similar to the process depicted in Fig. 1. So we leave it out in this paper. 


\section{Experiment: appraisal of chinese spirits}

We have developed a fuzzy ontology based on ef$S \mathcal{H I N}$ and applied it to the appraisal of Chinese spirits. Here, we just give a FCBR example and omit most other concepts and roles due to space limitations.

Chinese spirits can be categorized as Maotaiflavor, Luzhou-flavor, Fen-flavor, Rice flavor and Other-flavor spirits. The aroma evaporated from Chinese spirits can also be further partitioned into soy sauce, heavy, light, sweet and other aroma. It is well known that there is a close relation between spirits and aroma. So, this relation is essentially of typical FCBR, we call it $\mathrm{R}_{\mathrm{s}-\mathrm{a}}$.

According to the reference process in Fig. 1, it should hold when assigning membership degrees for FCBR $R_{s-a}$ : (1) triple $<$ Spirits, aroma, "FCBR" $>\in Q$, (2) set $\{<$ Spirits, Maotai-flavor $>,<$ Spirits, Luzhouflavor $>$, <Spirits, Fen-flavor $>,<$ Spirits, Rice-flavor $>$, $<$ Spirits, Other-flavor $>\} \subseteq \mathrm{R}_{\mathrm{kf}}$ and (3) set $\{<$ Aroma, Soy-sauce $>$, <Aroma, Heavy $>$, <Aroma, Light>, $<$ Aroma, Sweet $>,<$ Aroma, Other-aroma $>\} \subseteq \mathrm{R}_{\mathrm{kf}}$. Then, the process builds a matrix and presents it to Chinese spirits experts who are responsible for specifying membership degrees for $\mathrm{R}_{\mathrm{s}-\mathrm{a}}$. After this done, the process turn the matrix into a group of fuzzy concept relations: \{<Maotai-flavor, Soy-sauce, 0.95>, $<$ Maotai-flavor, Heavy, $0.8>, \ldots,<$ Other-flavor, Other aroma, $0.9>$ \}.

The full membership degrees of all pairs in $\mathrm{R}_{\mathrm{s}-\mathrm{a}}$ are shown in Table 2. The degrees indicate, for example, that a distilled spirit with soy-sauce aroma belongs to Maotai-flavor spirit with $95 \%$ probability. Note that each pair represents one fuzzy concept relation.

\begin{tabular}{cccccc}
\hline Spirits & Soy & Heavy & Light & Sweet & Other \\
\hline Maotai & 0.95 & 0.8 & 0.4 & 0.5 & 0.5 \\
Luzhou & 0.8 & 0.95 & 0.7 & 0.7 & 0.5 \\
Fen & 0.4 & 0.7 & 0.95 & 0.8 & 0.5 \\
Rice & 0.5 & 0.7 & 0.8 & 0.95 & 0.5 \\
Other & 0.5 & 0.5 & 0.5 & 0.5 & 0.9 \\
\hline Table 2: & The FCBR relation between spirits and aroma.
\end{tabular}

\section{Discussion and conclusions}

In this paper, we have proposed a reasoning-enabled general fuzzy ontology extending domain ontology to involve uncertainty knowledge. Besides the wellknown fuzzy concepts, the general fuzzy ontology categorizes fuzzy information in ontology applications, based on our investigation, into fuzzy instance relation, fuzzy concept relation, and fuzzy concept base relation.
Furthermore, this paper has also drawn an analysis on the general fuzzy ontology in a description logic view, bringing out a fuzzy description logic ef-SHIIN. At last but not the least, we suggested reference processes for building these three fuzzy relations and closed this paper with a case to which the general fuzzy ontology was applied.

However, there are still some issues to be further studied. The gathering of membership degrees is a still tedious and time-consuming work. Practitioner should employ appropriate mathematic functions to do such job as much as possible. In addition, though the three fuzzy relations can be easily transplanted to other FDLs, reasoning on FDLs with concrete domain is still unavailable so far. It is the biggest impediment to the popular use of the general fuzzy ontology. Moreover, evolution of ontology is still an untouched field in general fuzzy ontology.

\section{Acknowledgement}

This work is partially supported by the National Basic Research Program (973) of China (Grant No. 2003CB317000), the Science and Technology Plan of Zhejiang Province (Grant No. 2007C33072).

\section{References}

[1] I. Horrocks, U. Sattler, S. Tobies, Practical reasoning for expressive description logics. Proceedings of the 6th International Conference on Logic for Programming and Automated Reasoning (LPAR'99), Number 1705 in LNAI, pp. 161-180, 1999.

[2] C.S. Lee, Z.W. Jian, L.K. Huang, A Fuzzy Ontology and Its Application to News Summarization. IEEE Transactions on Systems, Man and Cybernetics, 35: 859-880, 2005.

[3] J.J. Lu, B.W. Xu, Y.H. Li and D.Z. Kang, et.al., Extended fuzzy ALCN and its tableau algorithm. Proceedings of the Second International Conference on Fuzzy Systems and Knowledge Discovery, pp. 232-242, 2005.

[4] D.Z. Kang, B.W. Xu, J.J. Lu and Y.H. Li, Reasoning for a fuzzy description logic with comparison expressions. Proceedings of the 2006 International Workshop on Description Logics, pp. 111-118, 2006.

[5] T.T. Quan, C.H. Siu, T.H. Cao, Automatic Fuzzy Ontology Generation for Semantic Web, IEEE Transactions on Knowledge and Data Engineering, 18: 842 - 856, 2006. 
[6] U. Straccia, Reasoning within fuzzy description logics. Journal of Artificial Intelligence Research, 14: 137-166, 2001.

[7] U. Straccia, Towards a fuzzy description logic for the semantic web: preliminary report. Proceedings of ESWC, pp. 167-181, 2005.

[8] G. Stoilos, G. Stamou, V. Tzouvaras and J.Z. Pan, et.al., The fuzzy description logic f-SHIN, Proceedings of the International Workshop on Uncertainty Reasoning for the Seantic Web, pp. 67-76, 2005.

[9] L.A. ZADEH, Fuzzy sets, Information and Control, 8: 338 -353, 1965. 